\title{
TMS Perturbs Saccade Trajectories and Unmasks an Internal Feedback Controller for Saccades
}

\author{
Minnan Xu-Wilson, ${ }^{1}$ Jing Tian, ${ }^{2}$ Reza Shadmehr, ${ }^{1,3}$ and David S. Zee ${ }^{2,3}$ \\ ${ }^{1}$ Department of Biomedical Engineering, ${ }^{2}$ Department of Neurology, and ${ }^{3}$ Department of Neuroscience, The Johns Hopkins School of Medicine, Baltimore, \\ Maryland 21205
}

When we applied a single pulse of transcranial magnetic stimulation (TMS) to any part of the human head during a saccadic eye movement, the ongoing eye velocity was reduced as early as $45 \mathrm{~ms}$ after the TMS, and lasted $\sim 32 \mathrm{~ms}$. The perturbation to the saccade trajectory was not due to a mechanical effect of the lid on the eye (e.g., from blinks). When the saccade involved coordinated movements of both the eyes and the lids, e.g., in vertical saccades, TMS produced a synchronized inhibition of the motor commands to both eye and lid muscles. The TMS-induced perturbation of the eye trajectory did not show habituation with repetition, and was present in both pro-saccades and anti-saccades. Despite the perturbation, the eye trajectory was corrected within the same saccade with compensatory motor commands that guided the eyes to the target. This within-saccade correction did not rely on visual input, suggesting that the brain monitored the oculomotor commands as the saccade unfolded, maintained a real-time estimate of the position of the eyes, and corrected for the perturbation. TMS disrupted saccades regardless of the location of the coil on the head, suggesting that the coil discharge engages a nonhabituating startle-like reflex system. This system affects ongoing motor commands upstream of the oculomotor neurons, possibly at the level of the superior colliculus or omnipause neurons. Therefore, a TMS pulse centrally perturbs saccadic motor commands, which are monitored possibly via efference copy and are corrected via internal feedback.

\section{Introduction}

The motor commands that initiate a saccadic eye movement to a given target location exhibit variability as reflected, for example, in peak velocity. Motor commands depend on the content of the stimulus (Xu-Wilson et al., 2009b), the reward associated with the stimulus, the predictability of the stimulus (Straube et al., 1997; Golla et al., 2008; Xu-Wilson et al., 2009a), and whether or not the stimulus is also the target of a reaching movement (Epelboim et al., 1997). Despite this variability in the commands that initiate the movement, the saccade generally arrives on target. Because the oculomotor commands do not rely on immediate visual or proprioceptive feedback during the saccade (Guthrie et al., 1983), it has long been hypothesized that the brain relies on an internal feedback mechanism that monitors ocular motor commands, estimates the position of the eyes, and corrects during the

Received March 29, 2011; revised June 25, 2011; accepted June 28, 2011.

Author contributions: M.X.W., J.T., R.S., and D.S.Z. designed research; M.X.W. and J.T. performed research; M.X.W. analyzed data; M.X.W., R.S., and D.S.Z. wrote the paper.

This work was supported by the National Center for Research Resources (NCRR), a component of the National Institutes of Health (NIH), and NIH Roadmap for Medical Research (Grant UL1 RR 025005). Its contents are solely the responsibility of the authors and do not necessarily represent the official view of NCRR or NIH. This work was also supported by grants from the NIH (NS37422 and EY01849), the Leon Levy Foundation, the Helena Rubenstein Foundation, and the Schwerin Family Foundation. M.X.W. was supported by National Research Service Award predoctoral fellowships from the National Institute of Neurological Disorders and Stroke. J.T. is the Betty and Paul Cinquegrana Scholar. We thank Dale Roberts and Adrian Lasker for their technical support. We thank Dr. Lance Optican for his valuable input and engaging discussions. The model in this paper was modified from that given to us by Dr. Stefano Ramat and Dr. Lance Optican.

Correspondence should be addressed to Dr. Minnan Xu-Wilson, Massachusetts Eye and Ear Infirmary, 243 Charles Street, 9th floor, Boston, MA 02114. E-mail: manon@alum.mit.edu.

DOI:10.1523/JNEUROSCI.1584-11.2011

Copyright $\odot 2011$ the authors $\quad 0270-6474 / 11 / 3111537-10 \$ 15.00 / 0$ ongoing saccade for factors that could influence the efferent command and cause the movement to deviate from its intended goal (Zee et al., 1976; Optican and Robinson, 1980; Quaia et al., 1999; $\mathrm{Xu}$-Wilson et al., 2009a). Testing this hypothesis would benefit from a noninvasive technique in which the saccade motor commands could be briefly perturbed, engaging the hypothetical internal feedback system to perform within-movement corrections. However, unlike reaching movements in which the process of state-estimation can be indirectly observed by quantifying the response to an external perturbation (Bhushan and Shadmehr, 1999; Izawa and Shadmehr, 2008), it is difficult to perturb ongoing saccades using noninvasive methods.

A few invasive studies in monkeys have demonstrated that saccades can be perturbed in flight (Keller and Edelman, 1994; Keller et al., 1996, 2000; Munoz et al., 1996). In humans, there are currently two techniques for perturbing a saccade: electrical stimulation of the face around the supraorbital nerve and a sudden noise burst (Becker, 1993; Goossens and van Opstal, 2010). Facial stimulation disrupts the trajectory of the eyes during a saccade, producing a pause in the saccade. A sudden noise can produce a blink that perturbs the trajectory of the eyes during a saccade. Unfortunately, these techniques have significant drawbacks, as facial stimulation is painful and loud noises are uncomfortable and show habituation (Koch, 1999).

Transcranial magnetic stimulation (TMS) is an increasingly popular tool in neuroscience. Its effects on the brain, however, are not well understood. Here, we report a novel and unexpected effect of TMS that is unrelated to the position of the TMS coil on the head. We found that when a single pulse is applied immediately before or during a saccade, it engages a startle-like neural 
reflex that briefly alters the ongoing oculomotor commands, slowing or even transiently stopping the eye movement. Despite this perturbation, the movement is corrected with commands that arrive later in the same saccade, accurately steering the eyes close to the target even when the target stimulus is no longer visible. Using simulations, we found that a saccade generation model with feedback control is consistent with many of the interesting features of TMS-perturbed saccades.

\section{Materials and Methods}

We tested five healthy control subjects (average age 42 years; three males and two females) as they performed visually guided saccades. All subjects gave written consent to protocols approved by the Johns Hopkins Institution Review Board.

Subjects sat in a dark room with their heads restrained with a dental bite-bar. We used a scleral search coil system to record, at $1000 \mathrm{~Hz}$, horizontal and vertical eye movements of either the right or the left eye, or in one subject from both eyes (Robinson, 1963). Eye position signals from the coils were filtered in hardware $(90$ $\mathrm{Hz}$ low-pass Butterworth), digitized $(1000 \mathrm{~Hz})$, and saved on a computer for later analysis. A third-order Savitzky-Golay filter was applied to the position signal to derive the velocity and acceleration signals. A $0.2^{\circ}$ red laser beam was rear-projected onto a translucent screen located $1 \mathrm{~m}$ in front of the subject. Target position was varied by a servo-controlled mirror.

While all data presented here were recorded from the magnetic search coil system, we also recorded eye movements using infrared video tracking (Eyelink 1000; SR Research) in a few additional subjects. This allowed us to confirm that the perturbing effects of TMS on saccade trajectories were not due to potential artifacts of recording using search coils. Furthermore, we recorded high-resolution $(500 \mathrm{~Hz}$ frame rate) video images of the eye in conjunction with measuring eye position using the coil system. This allowed us to have independent evidence to corroborate the perturbation of the movement of the eye that was induced by the TMS during saccades.

TMS was performed with a Magstim 200 stimulator with a maximum output of 2.2 tesla, connected to a figure-eight magnetic coil, each loop having a diameter of $7 \mathrm{~cm}$. The stimulation strength varied from $50 \%$ to $60 \%$ of 2.2 tesla. In initial experiments, the TMS coil was placed at various positions on the head using MRI-guided neuro-navigation (Brainsight). As we could elicit pauses with TMS stimulation over many brain areas, we focused on stimulating the vertex, or $\mathrm{Cz}$ according to the EEG $10-20$ system.

The duration of saccades was determined by a $16 \%$ speed threshold. Abnormal saccades were excluded from analysis using global criteria that were applied to all subjects, as follows: (1) saccade amplitude $<67 \%$ of the target displacement, ranging from $15^{\circ}$ to $30^{\circ}$; (2) saccade reaction time $<100$ $\mathrm{ms}$ or $>500 \mathrm{~ms}$; and (3) abnormal saccade trajectories due to large blinks.

Saccades perturbed by TMS showed various velocity profiles. Some decelerated briefly while others came to a complete stop. A saccade was labeled as "paused" when the velocity profile had two clear peaks (Fig. $1 B$ ). Pause velocity was the local minimum in the velocity profile following TMS. Here we only considered local minima of $<50^{\circ} / \mathrm{s}$. Pause duration was the period during which velocity remained within $16 \%$ of the local minimum (pause velocity). The end of the pause was also the beginning of the resumed movement. The end of the resumed movement was determined using a fixed velocity threshold $(16 \%)$.

Experiment 1: TMS at saccade onset

Pro-saccades. On each trial, subjects had to fixate on the target for a random period of $1500-2300 \mathrm{~ms}$, after which the target stepped $15^{\circ}$ or $30^{\circ}$ away. Horizontal, vertical, and oblique saccades were tested in separate blocks. TMS was triggered near saccade onset $(30 \%$ s velocity threshold) on randomly selected trials (probability 67\%). Later analysis showed that the earliest time we could trigger TMS was $\sim 5 \mathrm{~ms}$ into the saccade. This delay was due to online filtering of the velocity signal. We placed the TMS coil at various places on the head, including the cerebellum and parietal cortex. In three subjects, we also tested whether the sound of TMS was enough to perturb saccades by discharging the TMS coil at least $30 \mathrm{~cm}$ above the head. A typical experiment consisted of seven to nine sets with 48 saccades per set.

Anti-saccades. In two subjects, we considered the effect of TMS on both pro-saccades and anti-saccades. In pro-saccade blocks, subjects were instructed to look at the target. In the anti-saccade blocks, they were instructed to look to a point in the opposite direction of the target, but equal distance from fixation.

In some experiments, we blanked the target as soon as saccade onset was detected (Fig. 2). This ensured that the corrective movement that followed the TMS-induced perturbation was not visually guided. We also performed control experiments in two subjects to measure eyelid and head movements concurrently with eye movements during saccades. In these experiments, small search coils were placed on the upper eyelid to measure lid motion, on the forehead to measure head motion, and on the eyes to measure the saccade.

\section{Experiment 2: TMS during the saccade}

To examine how timing of TMS within a saccade affected trajectories, we considered oblique saccades of $30^{\circ}\left(21.2^{\circ}\right.$ in the horizontal and vertical components). This larger saccade was chosen to provide a larger window of time during which TMS could disrupt saccade trajectories. The TMS coil was always placed on the top of the head. TMS was randomly delivered near saccade onset ( $5 \mathrm{~ms}$ after saccade start) or at 15, 25, 35, 45 , or $55 \mathrm{~ms}$ into the saccade. TMS was delivered on randomly chosen trials $(70 \%$ of the trials). Because our TMS generator took $\sim 4 \mathrm{~s}$ to charge, the smallest intertrial interval was $\sim 4 \mathrm{~s}$. The average time between TMS pulses was $6.4 \mathrm{~s}$.

\section{Experiment 3: TMS before the saccade}

We also applied TMS at various times before the saccade. We used $15^{\circ}$ oblique saccades symmetric around the midline $\left(10.6^{\circ}\right.$ in horizontal and vertical components). We assumed a saccade latency of $180 \mathrm{~ms}$ and triggered TMS with respect to target onset randomly at 40,60 , or $80 \mathrm{~ms}$ before the expected saccade onset. We made minor adjustments of TMS timing during the experiment to ensure a good distribution of TMS times before saccade onset. For the postexperiment analysis, we grouped trials into bins according to the actual time of TMS before saccade onset. The 
A
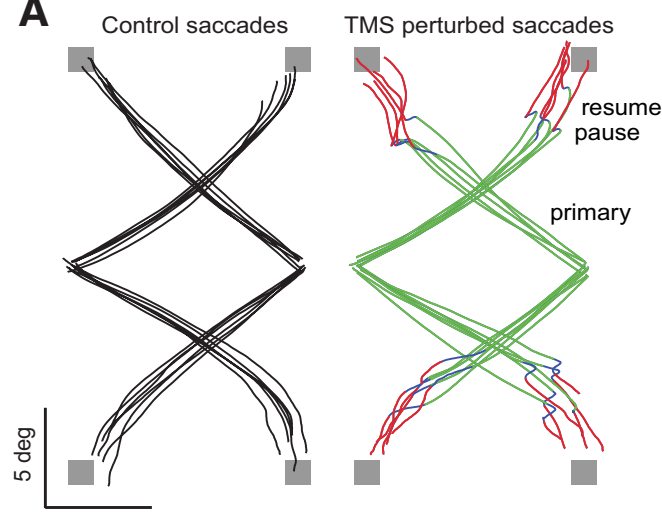

C

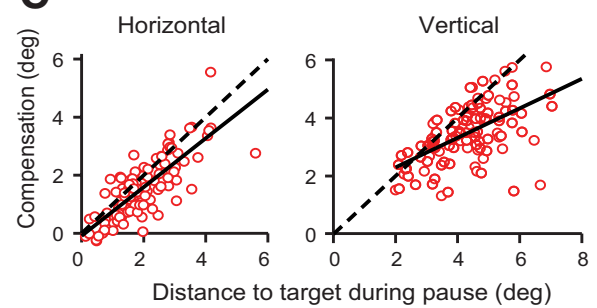

B
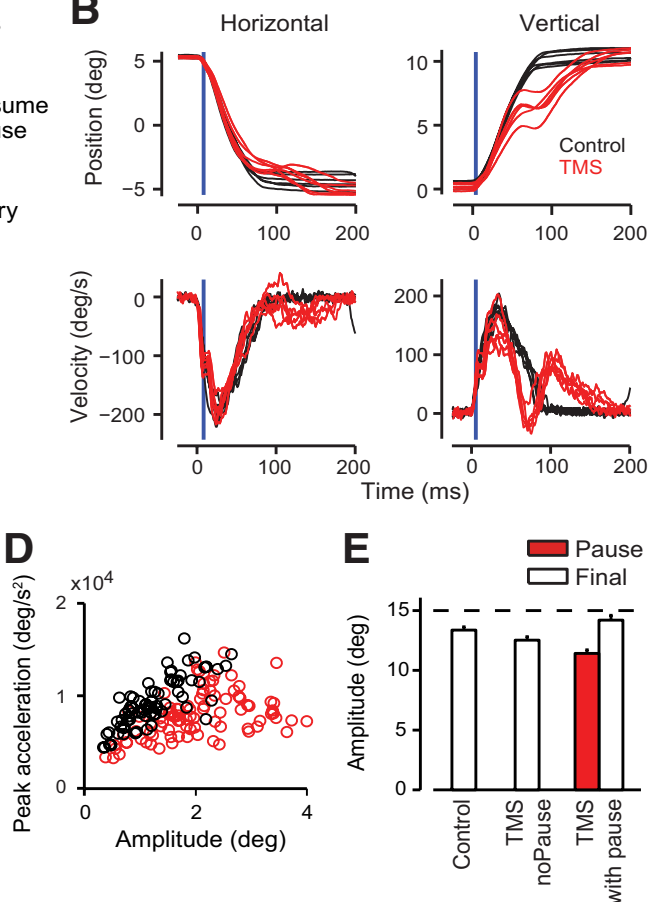

Figure 2. TMS-induced perturbations in oblique saccades. Data in $\boldsymbol{A}-\boldsymbol{D}$ are from a single subject and $\boldsymbol{E}$ shows group data. The target was turned off at target onset for these experiments, ensuring that compensation of the pause does not depend on visual input. $\boldsymbol{A}$, Two-dimensional trajectories of oblique saccades. For the TMS perturbed trials, the primary movement (green), the pause period (blue), and the compensatory movement (red) are highlighted. $\boldsymbol{B}$, Horizontal and vertical position and velocity traces of the same saccades as in $\boldsymbol{A}$. Note that peak velocity of vertical component of control saccades (no TMS) is less than that of the horizontal component. Blue vertical line denotes time of TMS. C, Compensation in the horizontal and vertical components for paused saccades. The size of the compensatory movement is highly correlated with the distance to target during the pause. Dotted lines denote perfect compensation of the error during the pause. Solid lines show the least mean square fit of actual compensation. $\boldsymbol{D}$, Peak acceleration of the compensatory movement. The compensatory movements (red) are often slower than saccades of comparable amplitude (black). These control saccades were taken from visually guided corrective saccades during the same experiment in the same subject. $\boldsymbol{E}$, Group data of saccade amplitude. The final amplitude of the paused saccades after the compensatory movement was larger than control saccades. There were some trials with TMS that did not show a pause (middle bar). These saccades had end positions that were closer to the target than saccades that showed a pause, suggesting that they did not show a correction because the eye was already close to the target. TMS coil was placed over C $z$ during acquisition of these data.

TMS coil was always placed at the top of the head. TMS was applied randomly on $75 \%$ of the trials with an average time of 5.5 s between TMS pulses.

\section{Neural modeling}

We tested a simple feedback control model of saccades (Ramat et al., 2005) to see how well it could describe emergent properties of saccades perturbed by TMS. The key components of the model are as follows: (1) two coupled excitatory burst neuron and inhibitory burst neuron pairs; (2) the burst neurons fired at a rate that depends on the size of the difference between the current estimate of eye position and the target position; (3) the motor error calculated by integrating the velocity output from the burst neurons and then subtracting this estimate of current eye position from the desired goal of the movement- the integration served as a state estimator, providing an ongoing internal feedback to the system; and (4) the burst neurons' membranes were modeled as high-pass filters with adaptation. This design simulated the postinhibitory rebound (PIR) of burst neuron membranes and allowed the burst neurons to fire immediately after the inhibition from the omnipause neurons (OPNs) was removed. The OPN of this model is simply a gate that is open when the motor error is higher than a threshold of $2^{\circ}$ (for detailed parameter values, see Ramat et al., 2005).

To compare feedback versus open-loop control of saccades, we inhibited the output of the burst generator either inside or outside of the feedback loop (Fig. 6A, i and ii, respectively). The effect of TMS was simulated as a drop in the burst neuron firing rate by 150 for $30 \mathrm{~ms}$. To simulate the effect of TMS by OPN reactivation (Fig. $6 A$, iii), we simply closed the OPN gate for $30 \mathrm{~ms}$ during the saccade to inhibit the burst neurons, while locations $i$ and ii were left unperturbed. The OPN to burst neuron gain [kept at 10 as in the original Ramat model (Ramat et al.,
2005)] determined the amount of inhibition and therefore the amount of postinhibitory rebound in the resumed movement. The feedback delay, presumably through the cerebellum, was set at $10 \mathrm{~ms}$ (Keller et al., 1983). All other aspects of the model remained unchanged.

\section{Results}

In experiment 1 , we found that in $74 \%$ of the trials, TMS perturbed the saccade trajectory regardless of location of stimulation on the head. Figure 1 shows examples of saccades to targets in the horizontal direction and Figure 2 shows saccades to targets in the oblique direction. The perturbation of the saccade was always in the form of a pause in the velocity profile. These pauses were nonspecific to the location of TMS, as we observed similar effects whether we applied TMS to the top of the head, over the cerebellum, over the parietal cortex, or over the frontal lobe (Fig. 3). The spatial nonspecificity of the effect produced by the TMS was particularly on display in the occasional trials in which we perturbed saccades by discharging the TMS coil at $30 \mathrm{~cm}$ above the head (Fig. 3). Antisaccades demonstrated pauses regardless of the stimulation location just as pro-saccades did (Fig. 3C).

The time delay from TMS to the local minimum in the velocity profile was $66 \pm 4 \mathrm{~ms}$ and the pause duration was $22 \pm 4 \mathrm{~ms}$. Even though pauses occurred $\sim 66 \mathrm{~ms}$ after TMS discharge, the effect of TMS in perturbing the eyes started earlier. A closer look at the velocity profiles showed that the interrupted saccades deviated from the control profile at $\sim 45 \pm 6 \mathrm{~ms}$ after time of TMS (Fig. 
A Horizontal pro-saccades in Subject 1

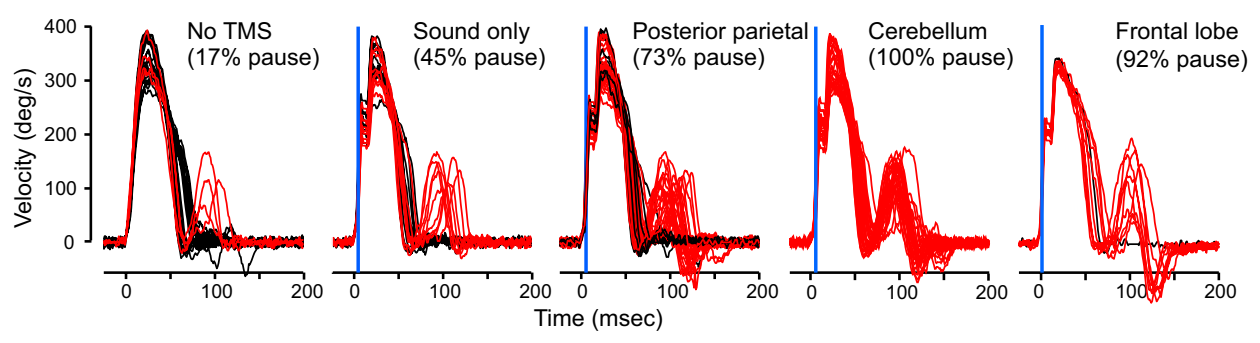

B Horizontal pro-saccades in Subject 2

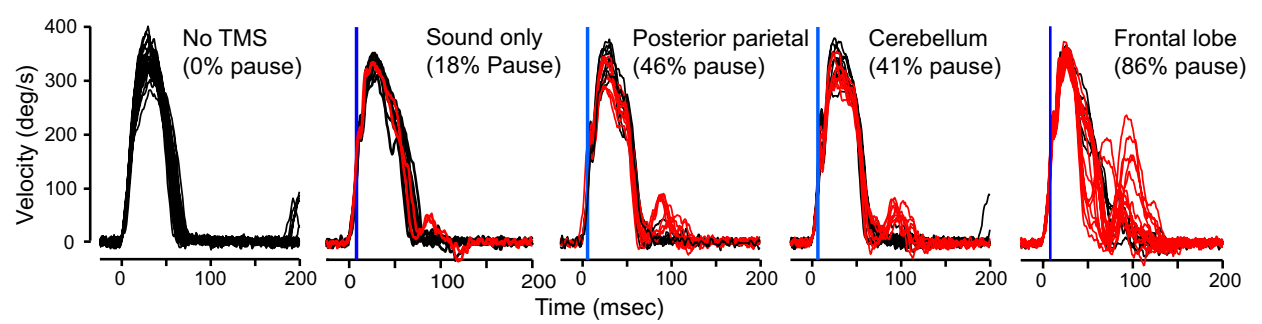

C Horizontal anti-saccades in Subject 2

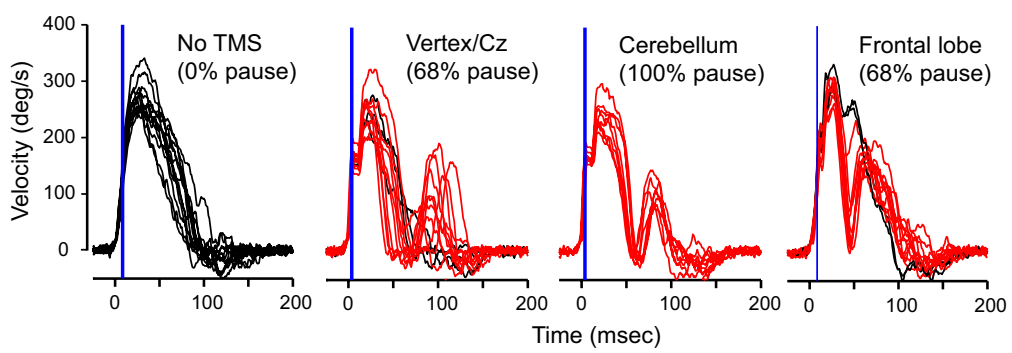

Figure 3. Lack of sensitivity to location of TMS. Data are from experiment 1 in which there was a $67 \%$ probability of TMS on a given trial. $A$, Data from one subject. Control saccades are in black, saccades that exhibited a pause are in red. Subject 1 exhibited some saccades that had a pause even when there was no TMS on that trial. In the sound-only trials, the TMS coil was placed $30 \mathrm{~cm}$ above the head. TMS applied over the parietal cortex and the cerebellum produced similar pauses. The small kink in the velocity profiles is an artifact of TMS. B, C, Data from a second subject showing pro-saccades and anti-saccades with TMS at various locations. This subject showed almost no pauses for the no TMS and sound-only conditions, whereas TMS at different locations on the head showed similar pauses in saccades. Cz refers to the top of the head in the EEG $10-20$ system. The velocity traces are for horizontal $15^{\circ}$ saccades.

$2 B)$. Thus, the inhibitory effect on saccades started $\sim 45 \mathrm{~ms}$ after TMS and persisted for $32 \mathrm{~ms}$ before the eyes reaccelerated. The pause was present in both the horizontal and the vertical components of saccades. For example, in oblique saccades there was no difference in the slowing-time of the horizontal and vertical components $(p=0.85)$ and there was a significant correlation in the pause time of the horizontal and vertical components $(r>0.4$ and $p<0.05$ ) for each of the four subjects in whom we measured oblique saccades. The effect of TMS was often more pronounced in the vertical component of oblique saccades (i.e., larger amplitude of resumed movement) (Fig. $2 B$ ). This is likely because the vertical component was typically slower than the horizontal component and therefore needed a larger correction to reach the target.

In an experiment in which we recorded from both eyes, we observed that the pauses were conjugate. The two eyes both paused $\sim 66 \mathrm{~ms}$ with a tight correlation in pause timing $(r=0.91$, $p<10^{-17}$ ).

We found no habituation in the saccade perturbations caused by TMS when the stimulation was applied to the head. The pauses occurred just as frequently early in the experiment as late in the experiment. Repeated-measure ANOVA showed no change in likelihood of saccades pausing with set number $(p=0.494)$ and no linear trend $(p=0.414)$.

\section{Compensation for the perturbation}

Despite the perturbation, the eyes were guided with subsequent motor commands that brought them close to the target. In analyzing the saccades that had paused, we found that the size of the compensatory movement was highly correlated with the remaining distance to the target (Fig. 2C). For the subject shown in Figure $2 C$, the correlation between size of the compensatory movement and distance to target was $r=0.79(p<0.001)$ in the horizontal direction and $r=0.59(p<0.001)$ for the vertical direction. As a group, the correlation was, on average, $r=0.79$ in the horizontal direction and $r=0.74$ in the vertical direction. That is, the movement that immediately followed the pause had the appropriate size and direction to accurately compensate for the perturbation.

In some experiments, we blanked the target as soon as saccade onset was detected (Fig. 2). This ensured that the corrective movement that followed the perturbation was not visually guided. We compared this with experiments in which the target was kept on during the entire saccade for four subjects. The visual condition made no statistical difference in the time it took for resumed movement to start (pause duration, $p=0.4620$, paired $t$ test) nor how well the resumed movement compensated for the error during the pause (accuracy of the final endpoint, $p=$ 0.1759 , paired $t$ test). Therefore, the compensatory motor com- 
mands that followed the perturbation did not rely on visual feedback.

The compensatory movement was slower than visually triggered saccades of the same amplitude. To compare the compensatory movement with control saccades, we considered the small saccades that sometimes follow the primary saccade [i.e., a control saccade that happens to fall short and is then followed by a second saccade (Fig. 1A)]. The secondary saccades occurred, on average, $284 \mathrm{~ms}$ after completion of the control saccade and were appropriate for comparison as they had similar amplitude as well as similar starting eye position. We found that the peak acceleration of the compensatory movement was smaller than most of the control saccades of the same amplitude (Fig. 2D). For each subject, a one-way analysis of covariance (ANCOVA) was conducted. The independent variable was peak velocity or peak acceleration (for oblique saccades, we used tangential peak velocity and tangential peak acceleration as the independent variables). Dependent variables were amplitudes of compensatory movement and control saccades. After adjusting for saccade amplitude, ANCOVA showed that the compensatory movement was significantly slower than control saccades for all subjects by $18 \%$ for peak velocity $(p<0.005)$ and $3.2 \times 10^{30} / \mathrm{s}^{2}$ for acceleration $\left(p<10^{-9}\right)$.

Without TMS, the saccade toward a visual target typically falls slightly short. However, we found that the final amplitude of a perturbed saccade was, on average, larger than a control saccade by $0.83^{\circ}(p<0.05$, two-tailed $t$ test) (Fig. $2 E)$. That is, whereas control saccades were slightly hypometric, the compensation that followed the perturbed saccades made the saccade less hypometric.

We labeled a saccade as being perturbed if, in response to TMS, the eye trajectory exhibited a pause. A minority (26\%) of TMS saccades did not show a pause. These TMS no-pause saccades exhibited two curious properties, as illustrated in Figure $2 E$ : (1) their amplitudes were generally smaller than control saccades by $0.84^{\circ}(p<0.05$, paired $t$ test $)$ and $(2)$ their amplitudes were generally $1.10^{\circ}$ larger than the initial amplitudes of saccades that paused and resumed ( $p<0.01$, paired $t$ test). We conjecture that many of the TMS no-pause saccades were in fact perturbed (as evidence by their smaller mean amplitude), but did not show a correction because the movement ended close enough to the goal so that an immediate correction was unnecessary (on average $\sim 2.5^{\circ}$ away).

\section{Spatial nonspecificity of TMS perturbations of saccades}

In preliminary experiments, we placed the TMS coil using MRI guided neuro-navigation. However, we soon realized that we could elicit pauses with TMS over many brain areas. Data of horizontal pro-saccades from two subjects are shown in Figure 3, $A$ and $B$. For all but one subject (Fig. $3 A$, subject 1), no-TMS saccades did not exhibit pauses (Fig. $3 B$, subject 2). Subject 1 had saccades that exhibited a pause in a small number of no-TMS trials (17\%). For this subject, placement of the TMS coil in air at $30 \mathrm{~cm}$ above the head (sound-only condition) increased the probability of pause to $45 \%$. However, this subject did not always show the same frequency of pauses in different sessions of the experiment. In some sessions, a particular stimulation site produced more pauses than other regions, such as with cerebellar stimulation in subject 1 (Fig. $3 A$ ). However, this was not consistent from session to session. Regardless of the variability among subjects and within subjects across different sessions, TMS applied over the parietal cortex, the cerebellum, frontal lobe, or the vertex all produced similar pauses (Fig. $3 A, B$ ) with statistically similar pause timings for all sites.

The lack of specificity of the TMS stimulation might be due to the widespread networks involved in generating reflexive saccades (so-called pro-saccades). We therefore tested anti-saccades, which are more voluntary in nature and especially reliant on the frontal lobe. We applied TMS over the vertex, cerebellum, and frontal lobe during anti-saccades (Fig. 3C), all of which produced clear pauses in saccades. Pauses in anti-saccades occasionally occurred slightly earlier (by as much as $20 \mathrm{~ms}$ ) compared with pro-saccades in this particular subject, but were no different from pro-saccades in other subjects.

\section{Eyelid and head movements during saccades}

What was the mechanism with which the TMS perturbed saccades? One possibility is that TMS led to an eye blink or head movement that altered the trajectory of the eyes. To test for this, we placed a small search coil on the upper eyelid or the forehead. In this way, we were able to record the motion of the globe and eyelid or head simultaneously.

Examples of lid motion during voluntary blinks are shown in Figure $4 \mathrm{~A}$. We can compare the amplitude of the lid displacement during these voluntary blinks to the lid motion that is occasionally produced due to TMS. For example, when TMS is applied during fixation, it occasionally produces a blink. When we applied TMS at random times during fixation, we found that in response to the first few pulses there was a blink (Fig. 4B). However, the TMS-induced blinks during fixation rapidly habituated within eight trials (Fig. $4 B$ ). Figure $4 C$ shows horizontal saccades of a subject. Little lid movement occurred with horizontal saccades (Fig. 4C, bottom, black lines). When a TMS pulse was applied and produced a saccade with a pause, the motion of the lid was indistinguishable from the movements of the lid in a control saccade. Occasionally, the lid did show a movement that may have been due to the TMS (Fig. 4C), but this movement was never as big as one that we observed in a voluntary blink (Fig. $4 A$ ). Therefore, during horizontal saccades, the application of TMS altered the trajectory of the eye but not the lid. This suggests that the perturbation to the eye trajectory was not due to a mechanical interaction with a TMS-induced lid movement or a blink.

In vertical saccades, we found further evidence for the idea that the perturbations to the eye trajectory were not due to a mechanical interaction with the eyelids. Unlike in horizontal saccades, during vertical saccades the eyelids move substantially, producing a coordinated movement that includes a lid saccade (Becker and Fuchs, 1988) as well as an eye saccade (Fig. 4D). When we applied TMS during vertical saccades, we saw a clear pause in both the motion of the eyes and the motion of the lids. Indeed, in $86 \%$ of trials in which the eye trajectory exhibited a pause, the lid trajectory also exhibited a pause. For TMS trials that did not show pauses in the eye trajectory, there were also no pauses in the lid trajectory. Following the pause, as the corrective movement brought the eyes to the target, the eyelids also exhibited a motion that resembled a corrective movement. In summary, in saccades for which there was a coordinated movement of the eyes and the lid, application of TMS produced a pause in both movements.

Despite the fact that subjects were using bite-bars, it is possible that TMS might have induced small head movements, which could, in principle, have caused the perturbation in eye trajectory. To check for this, we recorded head movements using a search coil. We found no evidence for motion of the head as a 
consequence of brain stimulation (for data for stimulation of the head over the cerebellum, see Fig. $4 E$ ). Therefore, perturbation of the eye trajectory was not due to TMS-induced head or eyelid motion.

Effect of varying the timing of TMS

A $15^{\circ}$ control saccade typically lasts $75 \mathrm{~ms}$. The pause that we observed in TMS perturbed saccades at a latency of $\sim 66 \mathrm{~ms}$ (with respective to time of TMS), meaning that the pause came near the end of the movement. We interpreted the pause as an inhibition of the ongoing ocular motor commands and wondered whether we would observe a similar inhibition at similar delays for saccades that had longer durations. Therefore, in experiment 2, we systematically varied the timing of the TMS pulse with respect to saccade onset for $30^{\circ}$ oblique saccades. Figure $5 \mathrm{~A}$ displays the effect of TMS on saccade velocities. We observed that, whereas TMS applied early in the saccade transiently slowed the eyes but did not produce a pause, TMS applied late in the saccade could stop the eyes. We quantified the effect of TMS by subtracting the eye velocity in perturbed trials from the eye velocity in the control trials. We found that the amount of perturbation to the velocity profile was similar at all TMS times. On average, TMS induced a $44.6^{\circ} / \mathrm{s}$ drop $(p<$ $0.05)$ in velocity from that of control saccades. The maximum drop in velocity occurred at $63.8 \mathrm{~ms}$ and started $\sim 45 \mathrm{~ms}$, and this timing stayed invariant for all TMS times tested. The final amplitude of interrupted saccades overshot by $1.1^{\circ}$ compared with uninterrupted saccades, but the amount of overshoot was not correlated with time of TMS.

We also explored the effect of TMS when it was applied before the onset of the saccade. In experiment 3 , subjects made $15^{\circ}$ oblique saccades while TMS was triggered with respect to target onset in such a way as to arrive before the expected onset of the saccade. We found that three of four subjects showed shorter saccade latency with earlier TMS (with a positive and significant correlation between saccade latency and time of TMS with respect to target onset, $p<$ $0.05)$. Maximum drop in latency of 24 ms occurred for the earliest TMS time. Decreased saccade latency is consistent with the finding that startle can speed up externally triggered saccades (Castellote et al., 2007). Two subjects showed higher saccade velocity with TMS arriving $60 \mathrm{~ms}$ before saccade onset $\left(\sim 30^{\circ} \% \mathrm{~s}\right.$ faster, but no change in amplitude). When TMS arrived $80 \mathrm{~ms}$ before saccade onset, the saccade appeared to be unperturbed. TMS at -40 ms slowed the saccade, and at $-20 \mathrm{~ms}$ or closer to saccade onset, the saccades paused.

\section{Model and simulations}

The perturbation of eye trajectories provided us with a new way to study feedback control processes during a saccade. The fact
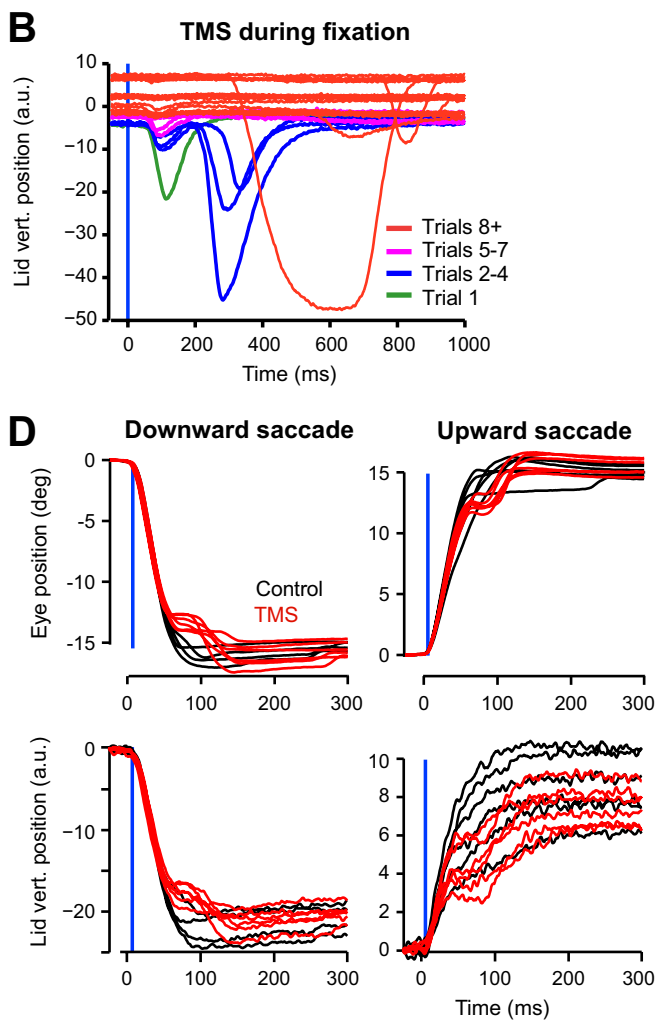

Lid and head motion. $\boldsymbol{A}$, Lid motion during voluntary blinks. Lid motion varies in duration but is generally much large

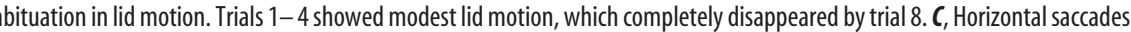
of lid motion caused by TMS is very small compared with voluntary blinks. D, During vertical saccades, the lid moves in a the eye and the lid. This is followed by a corrective movement in both structures. Blacklines are control trials (no TMS) and red lines are TMS trials that induced a pause. The blue vertical line indicates time of TMS. The TMS coil was placed over the cerebellum during acquisition of these data. $\boldsymbol{E}$, No head movement occurred during TMS. a.u., Arbitrary units; vert., vertical.

that perturbed saccades were corrected in flight suggests that an internal feedback system monitors the ocular motor commands, estimates the state of the eyes during a saccade, and corrects for potential perturbations. To test this idea, we used a previously published feedback control model of saccades (Ramat et al., 2005) and imposed perturbations on the motor commands at various nodes in the brainstem circuit. We imagined that TMS could have perturbed the oculomotor commands either within the feedback loop (feedback), in which the cerebellum could respond to the perturbation, or downstream of it (open-loop), in which the cerebellum could not have responded (Fig. 6A).

Figure $6 B$ shows simulations of open-loop versus feedback controller in response to TMS perturbations. We see that in the open-loop condition, disruption of the motor commands always produces a saccade that falls short of the control saccade. In contrast, in the feedback condition, the same disruption produces a perturbed saccade that is corrected by subsequent motor commands, bringing the eye to the control location. While it is not surprising that feedback can guide perturbed saccades to the tar- 
A

TMS after saccade onset

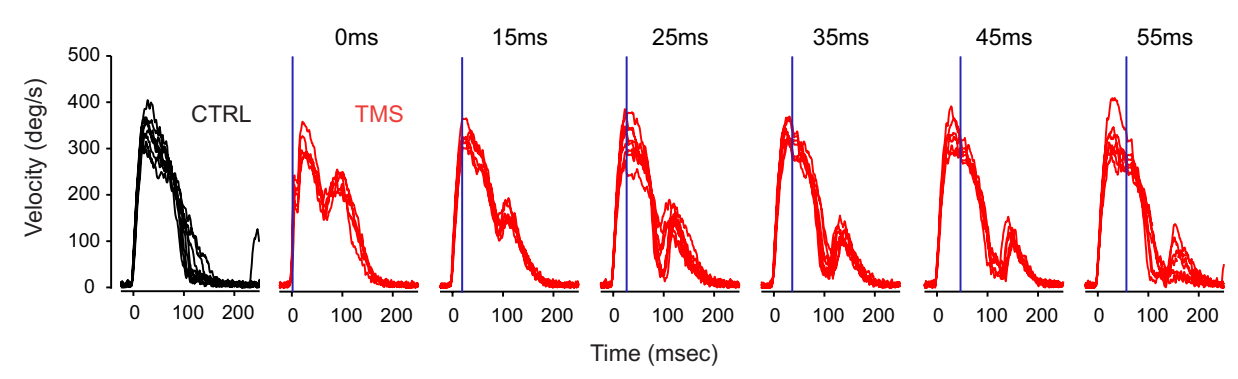

B

TMS before or at saccade onset

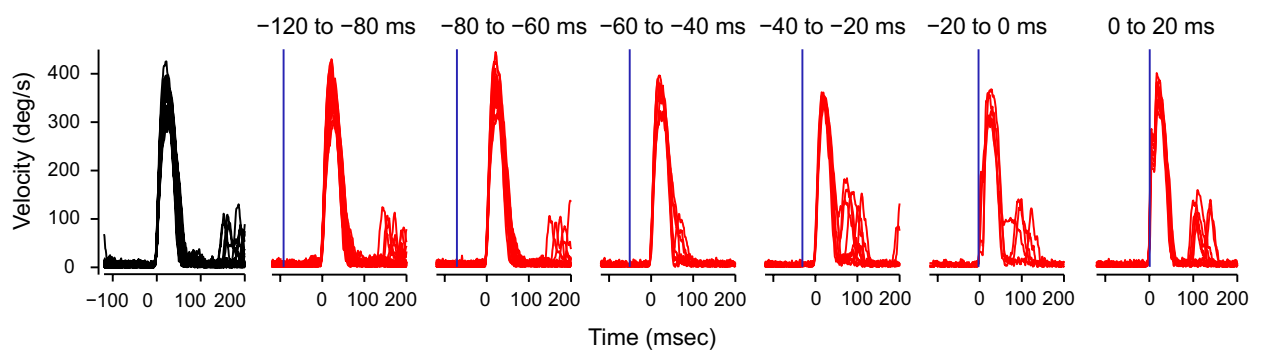

Figure 5. TMS applied at various times during saccades (experiments 2 and 3 ) for representative subjects. Plots show sample velocity traces. $A$, TMS applied at various times during $30^{\circ}$ oblique saccades. TMS applied early in the saccade slowed the eyes. TMS applied late in the saccade could completely stop the eyes. Maximum perturbation from TMS occurred $\sim 65 \mathrm{~ms}$ after the time of TMS. Vertical blue line indicates time of TMS. CTRL, Control. $\boldsymbol{B}$, TMS applied at various times before a $15^{\circ}$ oblique saccade. TMS at -120 to $-60 \mathrm{~ms} \mathrm{did} \mathrm{not} \mathrm{interrupt} \mathrm{saccades.} \mathrm{TMS} \mathrm{at}-40 \mathrm{~ms}$ slowed the saccade, and at $-20 \mathrm{~ms}$ or closer to saccade onset it paused it. TMS coil was placed over C $z$ during acquisition of these data.

get, a feedback control system with an accuracy criterion explains why some TMS trials in our data produced hypometric saccades that were not followed by a compensatory movement. These saccades were likely already close enough to the target and therefore did not reach threshold to trigger a correction. Our simulations support the idea that a feedback process is constantly checking the progress of the saccade commands against an internal accuracy criterion and actively compensating for perturbations like those induced by TMS.

The Ramat model (Ramat et al., 2005) nicely explains another interesting feature of our data: resumed movements are slower compared with normal saccades of comparable size. Slower resumed movements can be related to the hypothesized PIR property of burst neuron membranes. PIR has been proposed to be a property of saccade burst neurons and contribute to the initial saccade burst when prolonged inhibition from the OPNs is released (Enderle, 2002; Miura and Optican, 2006). Without this prolonged inhibition, the burst neurons do not fire as vigorously for the resumed movement. Our simulations showed that with PIR as a property of burst neurons, the corrective movement had a lower peak acceleration compared with normal saccades of the same size. Without PIR, resumed movements and normal saccades of the same size had the same peak acceleration (Fig. 6C).

Studies in experimental animals have been able to induce pauses in saccades by activating OPNs during saccades, thereby inhibiting output of the burst neurons (Keller et al., 1996). OPNs could be reactivated as a result of inhibition of the caudal superior colliculus by the trigeminal system (Goossens and Van Opstal, 2000) or perhaps by activation of other pathways to the OPN (Langer and Kaneko, 1990). We simulated OPN reactivation during saccades and found that saccades paused and resumed much like interrupted saccades produced by lowering burst neuron firing rates (Fig. $6 \mathrm{~B}$ ), but with one key difference. Simulation of pauses with OPN reactivation produced interrupted saccades with final positions that were hypermetric compared with unin- terrupted saccades (Fig. 6D), which is in agreement with another interesting feature found in our data. This is because during the pause period, the burst neurons are inhibited by reactivated OPNs, resulting in more vigorous firing of the burst neurons after the pause period (though not as vigorous as for saccades of the same size made after a normal prior fixation period). The inherent delay in the feedback loop does not allow for complete compensation of this overshoot. In this way, we can account for the seeming paradox that resumed saccades are slower than normal saccades of comparable size and yet are still hypermetric compared with uninterrupted saccades. In summary, many interesting features of the data (some perturbed saccades do not resume, hypermetricity in paused saccades, and slower resumed movements after the pauses), although seemingly random at first glance, are actually consistent with a feedback control model of saccade generation.

\section{Discussion}

A single TMS pulse applied to the head inhibited ongoing saccades with a delay of $45 \mathrm{~ms}$ and lasted $32 \mathrm{~ms}$. This inhibitory effect was followed by motor commands that accurately compensated for the perturbation and brought the eyes to the target. TMS perturbed both pro-saccades and anti-saccades. The location of TMS was not critical, as we observed similar effects when TMS was applied on top of the head, over the cerebellum, or over the parietal cortex. Thus, we have discovered a novel way to disturb premotor oculomotor commands and unmask an internal feedback control system.

\section{TMS may engage a startle-like circuitry}

The effect of TMS was not through generation of a blink, as lid movements did not correlate with eye perturbations during horizontal saccades (Goossens and van Opstal, 2010). Furthermore, we found that in vertical saccades, TMS produced a pause in both the motion of eye and lid, removing the possibility of lid motion 
causing a pause in the eye motion. Nor was the effect of TMS through direct excitation of the extraocular muscles or head motion, as this or any other kind of mechanical perturbation of the eye plant would not allow for in-flight compensation. Cocontraction could be another TMS effect but would be unlikely to stop the saccade.

The TMS-induced pauses are qualitatively similar to those induced by electrical stimulation of OPNs (Keller et al., 1996) or stimulation of rostral superior colliculus (Munoz et al., 1996; Gandhi and Keller, 1999), in that interrupted saccades are followed by a resumed movement, but the final amplitude tends to be hypermetric compared with control saccades. Therefore, it seems possible that TMS engages a circuit that affects the OPN and/or the superior colliculus.

Similar pauses in saccades have been observed with sudden sound and light stimuli, air-puffs, or surface electrical stimulation of the supraorbital nerve (Evinger et al., 1982; Becker, 1993; Goossens and van Opstal, 2010). The sound of TMS alone can engage the startle system (VallsSolé et al., 1999). In fact, magnetic stimulation of the supraorbital nerve has been suggested as a way of studying the startle system (Bischoff et al., 1993). We found that the sound of TMS alone could perturb saccades. However, the perturbation was more robust when the TMS was applied directly to the head. This is consistent with Becker's (1993) observation that stimulation of the supraorbital nerve was more effective in pausing saccades than noise or light. It seems plausible that TMS on the head produced a synchronized multimodal activation of the startle system, as stimuli that produce synchronized tactile, acoustic, and vestibular activations engage the startle system more strongly (Yeomans et al., 2002).

The saccade and startle systems overlap in circuitry. The reticular formation in the brainstem, critical in generating the startle response, houses the saccade burst generators (Büttner-Ennever and Horn, 2004) and receives acoustic, tactile, and vestibular inputs (Yeomans et al., 2002). The superior colliculus (SC), another part of the saccade circuitry, receives afferents from many regions including the sensory trigeminal nucleus, and stimulation of different regions in the SC can elicit orienting responses and startle reflexes (Dean et al., 1989; Lin et al., 2002). TMS could be perturbing saccades by altering the activity in the reticular formation and/or the superior colliculus.

Another reason to suspect engagement of the startle system is that its circuitry is not specific to the oculomotor system, but projects to all motor areas (Carlsen et al., 2011). Indeed, we found that TMS induced a synchronized inhibition of the motor commands to both the eye and lid muscles in vertical saccades. Siebner et al. (1999) showed that TMS has an inhibitory effect on the ongoing motor commands to the hand at a latency of $40 \mathrm{~ms}$, and this effect is present whatever part of the head is stimulated. The
B Open-loop versus feedback control of saccades
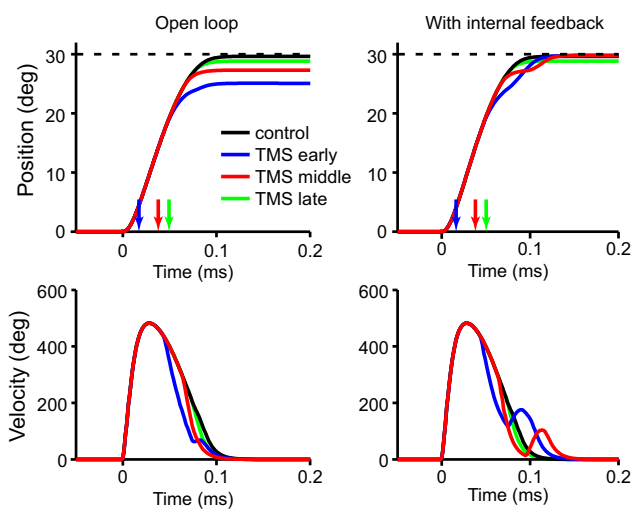

C Postinhibitory Rebound

D OPN reactivation
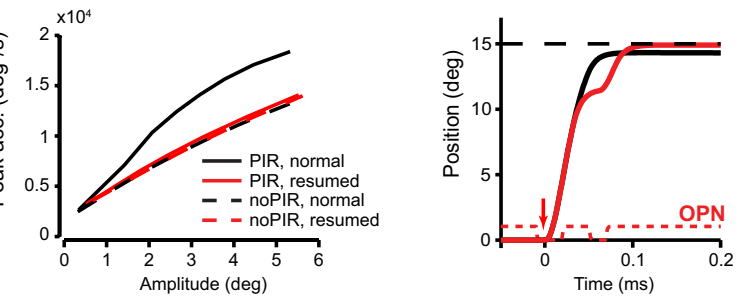

Figure 6. Model and simulations. $\boldsymbol{A}$, Schematic diagram showing feedback control of saccades in the Ramat model (Ramat et . 2005). OPNs gate the activity of the burst neurons allowing them to fire if an error is larger than a threshold. An estimator, postinhibitory rebound. The filled red circles indicate sites of TMS effect used in the simulations. $B$, Comparison of open-loop and feedback control of saccades in response to a pause in the saccade. Open-loop control predicts that interrupted saccades always fal car saccades shows that early TMS can slow the eyes and late TMS can cause the eyes to completely pause. Arrows indicate time in saccades, the resumed movements have an extra boost due to postinhibitory rebound, making the final endpoint of interrupted saccades larger than unperturbed saccades.

synchronized inhibitory effect of TMS on ongoing hand, eye, and lid motion, and its spatial nonspecificity, are consistent with the possibility that a single TMS pulse engages a startle-like reflex that inhibits ongoing motor commands.

We observed no habituation in response to TMS, contrary to Becker's (1993) finding that saccades interrupted by sound, light, and supraorbital stimulation all showed some habituation. This is surprising as habituation is a common feature of the startle reflex. However, startle can show rapid or little habituation depending on the stimulus. For example, startle in response to sound and light shows more rapid habituation than in response to tactile stimuli such as air puffs (Geyer and Swerdlow, 1998). The lack of habituation in TMS may be due to its multimodal nature, or applying TMS during a reaction time task may decrease habituation (Carlsen et al., 2003). A feature of startle that remains to be tested is prepulse inhibition, which predicts that a startle stimulus given within 30-120 ms before the TMS pulse would remove its inhibitory effect on saccades (Braff et al., 2011).

\section{TMS unmasks a feedback controller}

The perturbation induced by TMS was followed by motor commands that compensated and brought the eyes near the target. Our results provide strong evidence in favor of the hypothesis 
that saccades are not open-loop, but rather rely on internal feedback that continuously monitors the outgoing motor commands and estimates the current state of the eyes. Robinson proposed such an internal feedback loop and a number of investigators have suggested that the cerebellum contains a forward model, using efference copy of ocular motor commands to perform state estimation (Robinson, 1975; Quaia et al., 1999) and may then send this information to other oculomotor regions such as the superior colliculus (Arai et al., 1999; Soetedjo et al., 2002). Our earlier work demonstrated that natural variability in motor commands that initiate saccades are present in both healthy and cerebellar subjects, but whereas in healthy subjects this variability is corrected with commands that arrive later in the same saccade, in cerebellar patients the compensatory motor commands are missing (Xu-Wilson et al., 2009a). This framework predicts that cerebellar patients will have reduced or inaccurate compensatory motor commands in response to TMS-induced perturbations of saccades, a prediction that remains to be tested.

Our modeling points to feedback control and PIR as explanations of how interrupted saccades can sometimes be hypometric and other times hypermetric (if a resumed movement takes place). PIR is a property of some cell types that, at the offset of hyperpolarization, produces a rebound discharge mediated by low-threshold $\mathrm{Ca}^{2+}$ channels (Perez-Reyes, 2003). PIR has been proposed to be a property of saccade burst neurons and to contribute to the saccade burst after prolonged inhibition from the OPNs (Enderle, 2002; Miura and Optican, 2006). Diminished inhibition during the pause period would lead to less vigorous firing of the burst neurons during the resumed movement and render them slower compared with comparably sized saccades that occur after a normal period of fixation. Such a phenomenon may account for the slowing of saccades when they are made under closed eye (Shaikh et al., 2010), as OPNs remain off during sustained eye lid closure. In turn, burst neurons would not be hyperpolarized to the usual degree during fixation so that when the saccade begins PIR is less and the saccade is slower. Another reason why resumed movements could be slower than normal saccades is that they are memory driven rather than visually driven. To examine this possibility, we compared normal corrective saccades made in the dark versus those made with the target on (up to $4^{\circ}$ in amplitude) and found no significant difference in the relationship of saccade amplitude to peak velocity. It could also be that corrective movements driven by feedback mechanisms alone are slower, without the benefit of the full entourage of signals driving saccade burst neurons within the brainstem. Clearly, neurophysiological studies are needed to clarify exactly how corrective movements are generated.

\section{The use of TMS in studying functions of the brain}

TMS is generally viewed as a tool to disrupt the neural circuitry near the stimulation site. Our results paint a more complex picture and force us to reinterpret some earlier TMS studies, as well as serve as a caveat when interpreting the effects of TMS on motor and even cognitive behaviors. For example, Hashimoto et al. (1995) reported that TMS of the cerebellar vermis produced hypermetric ipsilateral and hypometric contralateral saccades. We systematically applied TMS to the back of the head, which presumably stimulated various parts of the cerebellar vermis. However, we did not observe any consistent effects to suggest that cerebellar function had been altered, although our stimulation may not have reached the $3-4 \mathrm{~cm}$ depth required to stimulate the cerebellar vermis. Rather, the only difference in applying TMS to the cerebellum was that the pauses occurred more frequently in one subject. Perhaps TMS on the back of the head more strongly engaged the startle pathways (Yeomans et al., 2002). Hashimoto and Ohtsuka (1995) did not report any pauses, though some saccades reversed direction briefly, which could indicate a TMSinduced perturbation. Thus, the nonspecific TMS-induced startle response is a potential confound when TMS is used to study the function of the brain.

\section{References}

Arai K, Das S, Keller EL, Aiyoshi E (1999) A distributed model of the saccade system: simulations of temporally perturbed saccades using position and velocity feedback. Neural Netw 12:1359-1375.

Becker W (1993) Banging the bang-bang circuit: experimental test of Robinson's local feedback theory of saccade generation. In: Contemporary ocular motor and vestibular research: a tribute to David A. Robinson (Fuchs AF, Brandt T, Buttner U, Zee DS, eds), pp 496-503. New York: Thieme.

Becker W, Fuchs AF (1988) Lid-eye coordination during vertical gaze changes in man and monkey. J Neurophysiol 60:1227-1252.

Bhushan N, Shadmehr R (1999) Evidence for learning of a forward dynamic model in human motor control. In: Advances in neural information processing systems 11 (Kearns MS, Kearns MS, eds). Cambridge, MA: MIT.

Bischoff C, Liscic R, Meyer BU, Machetanz J, Conrad B (1993) Magnetically elicited blink reflex: an alternative to conventional electrical stimulation. Electromyogr Clin Neurophysiol 33:265-269.

Braff DL, Geyer MA, Swerdlow NR (2011) Human studies of prepulse inhibition of startle: normal subjects, patient groups, and pharmacological studies. Psychopharmacology 156:234-258.

Büttner-Ennever JA, Horn AK (2004) Reticular formation: eye movements, gaze, and blinks. In: The human nervous system (Paxinos G, Mai JK, eds), pp 479-510. Amsterdam: Elsevier.

Carlsen AN, Chua R, Inglis JT, Sanderson DJ, Franks IM (2003) Startle response is dishabituated during a reaction time task. Exp Brain Res 152:510-518.

Carlsen AN, Maslovat D, Lam MY, Chua R, Franks IM (2011) Considerations for the use of a startling acoustic stimulus in studies of motor preparation in humans. Neurosci Biobehav Rev 35:366-376.

Castellote JM, Kumru H, Queralt A, Valls-Solé J (2007) A startle speeds up the execution of externally guided saccades. Exp Brain Res 177:129-136.

Dean P, Redgrave P, Westby GW (1989) Event or emergency? Two response systems in the mammalian superior colliculus. Trends Neurosci $12: 137-147$

Enderle JD (2002) Neural control of saccades. In: The brain's eye: neurobiological and clinical aspects of oculomotor research (Progress in brain research) (Hyönä J, Munoz DP, Heide W, eds), pp 21-49. Oxford: Elsevier.

Epelboim J, Steinman RM, Kowler E, Pizlo Z, Erkelens CJ, Collewijn H (1997) Gaze-shift dynamics in two kinds of sequential looking tasks. Vision Res 37:2597-2607.

Evinger C, Kaneko CR, Fuchs AF (1982) Activity of omnipause neurons in alert cats during saccadic eye movements and visual stimuli. J Neurophysiol 47:827-844.

Gandhi NJ, Keller EL (1999) Activity of the brain stem omnipause neurons during saccades perturbed by stimulation of the primate superior colliculus. J Neurophysiol 82:3254-3267.

Geyer MA, Swerdlow NR (1998) Measurement of startle response, prepulse inhibition, and habituation. In: Current protocols in neuroscience (Crawley JN, Skolnick P, eds). New York: Wiley.

Golla H, Tziridis K, Haarmeier T, Catz N, Barash S, Thier P (2008) Reduced saccadic resilience and impaired saccadic adaptation due to cerebellar disease. Eur J Neurosci 27:132-144.

Goossens HH, Van Opstal AJ (2000) Blink-perturbed saccades in monkey. II. Superior colliculus activity. J Neurophysiol 83:3430-3452.

Goossens HH, van Opstal AJ (2010) Differential effects of reflex blinks on saccade perturbations in humans. J Neurophysiol 103:1685-1695.

Guthrie BL, Porter JD, Sparks DL (1983) Corollary discharge provides accurate eye position information to the oculomotor system. Science 221:1193-1195.

Hashimoto M, Ohtsuka K (1995) Transcranial magnetic stimulation over the posterior cerebellum during visually guided saccades in man. Brain 118:1185-1193. 
Izawa J, Shadmehr R (2008) On-line processing of uncertain information in visuomotor control. J Neurosci 28:11360-11368.

Keller EL, Edelman JA（1994） Use of interrupted saccade paradigm to study spatial and temporal dynamics of saccadic burst cells in superior colliculus in monkey. J Neurophysiol 72:2754-2770.

Keller EL, Slakey DP, Crandall WF (1983) Microstimulation of the primate cerebellar vermis during saccadic eye movements. Brain Res 288:131-143.

Keller EL, Gandhi NJ, Shieh JM (1996) Endpoint accuracy in saccades interrupted by stimulation in the omnipause region in monkey. Vis Neurosci 13:1059-1067.

Keller EL, Gandhi NJ, Vijay Sekaran S (2000) Activity in deep intermediate layer collicular neurons during interrupted saccades. Exp Brain Res 130:227-237.

Koch M (1999) The neurobiology of startle. Prog Neurobiol 59:107-128.

Langer TP, Kaneko CR (1990) Brainstem afferents to the oculomotor omnipause neurons in monkey. J Comp Neurol 295:413-427.

Lin C, Wan X, Zhao W, Ma C, Ma C, Gao Y, Zhou Y, Yeomans JS, Li L (2002) Enhancement of electrically evoked startle-like responses by tetanic stimulation of the superior colliculus. Neuroreport 13:1769-1773.

Miura K, Optican LM (2006) Membrane channel properties of premotor excitatory burst neurons may underlie saccade slowing after lesions of omnipause neurons. J Comput Neurosci 20:25-41.

Munoz DP, Waitzman DM, Wurtz RH (1996) Activity of neurons in monkey superior colliculus during interrupted saccades. J Neurophysiol 75:2562-2580.

Optican LM, Robinson DA (1980) Cerebellar-dependent adaptive control of primate saccadic system. J Neurophysiol 44:1058-1076.

Perez-Reyes E (2003) Molecular physiology of low-voltage-activated T-type calcium channels. Physiol Rev 83:117-161.

Quaia C, Lefèvre P, Optican LM (1999) Model of the control of saccades by superior colliculus and cerebellum. J Neurophysiol 82:999-1018.

Ramat S, Leigh RJ, Zee DS, Optican LM (2005) Ocular oscillations gener- ated by coupling of brainstem excitatory and inhibitory saccadic burst neurons. Exp Brain Res 160:89-106.

Robinson DA (1963) A method of measuring eye movement using a scleral search coil in a magnetic field. IEEE Trans Biomed Eng 10:137-145.

Robinson DA (1975) Oculomotor control signals. In: Basic mechanisms of ocular motility and their clinical implications (BachyRita P, Lennerstrand G, eds), pp 337-374. Oxford, UK: Pergamon.

Shaikh AG, Wong AL, Optican LM, Miura K, Solomon D, Zee DS (2010) Sustained eye closure slows saccades. Vision Res 50:1665-1675.

Siebner HR, Auer C, Roeck R, Conrad B (1999) Trigeminal sensory input elicited by electric or magnetic stimulation interferes with the central motor drive to the intrinsic hand muscles. Clin Neurophysiol 110:10901099.

Soetedjo R, Kaneko CR, Fuchs AF (2002) Evidence that the superior colliculus participates in the feedback control of saccadic eye movements. J Neurophysiol 87:679-695.

Straube A, Robinson FR, Fuchs AF (1997) Decrease in saccadic performance after many visually guided saccadic eye movements in monkeys. Invest Ophthalmol Vis Sci 38:2810-2816.

Valls-Solé J, Rothwell JC, Goulart F, Cossu G, Muñoz E (1999) Patterned ballistic movements triggered by a startle in healthy humans. J Physiol 516:931-938.

Xu-Wilson M, Chen-Harris H, Zee DS, Shadmehr R (2009a) Cerebellar contributions to adaptive control of saccades in humans. J Neurosci 29:12930-12939.

Xu-Wilson M, Zee DS, Shadmehr R (2009b) The intrinsic value of visual information affects saccade velocities. Exp Brain Res 196:475-481.

Yeomans JS, Li L, Scott BW, Frankland PW (2002) Tactile, acoustic and vestibular systems sum to elicit the startle reflex. Neurosci Biobehav Rev 26:1-11

Zee DS, Optican LM, Cook JD, Robinson DA, Engel WK (1976) Slow saccades in spinocerebellar degeneration. Arch Neurol 33:243-251. 